\title{
Decreased activity of RCAN1.4 is a potential risk factor for congenital heart disease in a Han Chinese population
}

\section{Dear Editor,}

Congenital heart disease (CHD) is a major birth defect worldwide. However, the aetiology of $\mathrm{CHD}$ remains unclear and the detailed molecular mechanisms underlying $\mathrm{CHD}$ pathogenesis have not been fully understood. The human regulator of calcineurin (RCAN1) gene is highly expressed in human hearts and brains (Fuentes et al., 1995) and composed of seven exons that are alternatively spliced and/or transcribed by differential promoters to produce different isoforms (Fig. 1A) (Yang et al., 2000). RCAN1.1 (NM_004414) and RCAN1.4 (NM_203418) are the two major isoforms differentially expressed in many tissues and cells. RCAN1.1 is constitutively expressed while the expression of RCAN1.4 is induced by diverse stimuli (Yang et al., 2000; Lange et al., 2004). RCAN1.4 negatively regulates NFAT-mediated transcription (Lange and Yutzey, 2006; Qin et al., 2006), which is an important regulator for early heart development (de la Pompa et al., 1998). Although SNPs rs149048873, rs193289374 and rs143081213 in the RCAN1.1 promoter were not associated with sporadic CHD (Guo et al., 2015; Li et al., 2015), the function of RCAN1.4 in $\mathrm{CHD}$ remains elusive.

To investigate if the RCAN1.4 plays a role in heart development, we evaluated the expression of the RCAN1.1 and RCAN1.4 proteins in E10.5 mouse embryonic hearts and human hearts. The results revealed that both the RCAN1.1 and RCAN1.4 were expressed in mouse embryonic hearts and human hearts (Fig. 1B). In addition, the RCAN1.4 protein was easily detected in embryonic mouse hearts at E10.5 but not in embryonic mouse heads at E10.5, while RCAN1.1 protein levels were similar between embryonic mouse hearts and heads (Figs. $1 \mathrm{~B}$ and S1A). These results demonstrate that not only RCAN1.4 is expressed in hearts, but also its expression is differentially regulated, at least during mouse heart development, suggesting that the expression level of RCAN1.4 might contribute to human CHD.

To evaluate the association of RCAN1 gene with human $\mathrm{CHD}$, we performed an association study of variants in RCAN1 with CHD. The $-2 \mathrm{~kb}$ of $5^{\prime}$-upstream regulatory regions, coding regions and $3^{\prime} \mathrm{UTR}$ s for RCAN1.1 and RCAN1.4 transcripts were first sequenced by target-capture sequencing in 412 blood samples of sporadic CHD patients and 213 matched controls from Shandong populations (Table S1). Eight SNPs with a minor allele frequency $(\mathrm{MAF})>0.05$ were identified within the $-2 \mathrm{~kb}$ of $5^{\prime}$ regulatory region of RCAN1.1 and RCAN1.4 transcripts. Three SNPs (rs765610, rs1248192 and rs36012405) and the other five (rs10550296, rs8133540, rs2243890, rs4816491 and rs2300385) were positioned in $5^{\prime}$ regulatory regions of RCAN1.1 and RCAN1.4 transcripts, respectively (Fig. 1A). We also identified one rare mutation (rs149533318, RCA$\mathrm{N} 1.4^{\mathrm{E} 25 \mathrm{~K}}$ ) in the coding region of RCAN1.4. Among them, only the genotype distribution of SNP rs2243890 $A>G$ (HGVS Name: NM_203418:c.-1712 A>G) located in RCAN1.4 promoter was normally different between the CHDs and control (Table S2). We observed the higher frequencies of $\mathrm{G}$ allele in $\mathrm{CHD}(\mathrm{MAF}=0.09)$ than control (MAF $=0.05)(\mathrm{OR}=1.98,95 \% \mathrm{Cl}=1.20-3.27, P=0.007$ in additive model; $\mathrm{OR}=2.11,95 \% \mathrm{Cl}=1.26-3.53, P=0.004$ in dominant model) (Table S2). To further confirm the validity of the association of rs2243890 $A>G$ with $C H D$, we not only verified the status of $r 2243890 A>G$ by re-sequencing a $0.5-\mathrm{kb}$ genomic sequence around the SNP in the previously analysed 412 sporadic CHD patients and 213 matched controls, but also examined the same sequence in 458 newly collected sporadic CHD patients and 1,107 healthy controls from Shanghai by Sanger sequencing. Consistent results were obtained for this SNP (OR $=1.57,95 \%$ $\mathrm{Cl}=1.19-2.07, P=0.002$ ) (Table 1). Subsequently, we combined the data from both cohorts for combined analysis and the result showed that the distribution of the minor $\mathrm{G}$ allele of rs2243890 was significantly different between the $\mathrm{CHD}$ and control groups. The $\mathrm{CHD}$ risk was significantly increased by 1.66 -fold due to the $G$ allele (OR $=1.66,95 \%$ $\mathrm{Cl}=1.30-2.11, P=4.68 \mathrm{E}-05)$ (Table 1). The frequencies of all genotypes among the control subjects were in accordance with the Hardy-Weinberg expectation $(P>0.05)$. The statistical power of the study is 0.924 at the 0.05 significance level, which is calculated with the power and sample size 
A

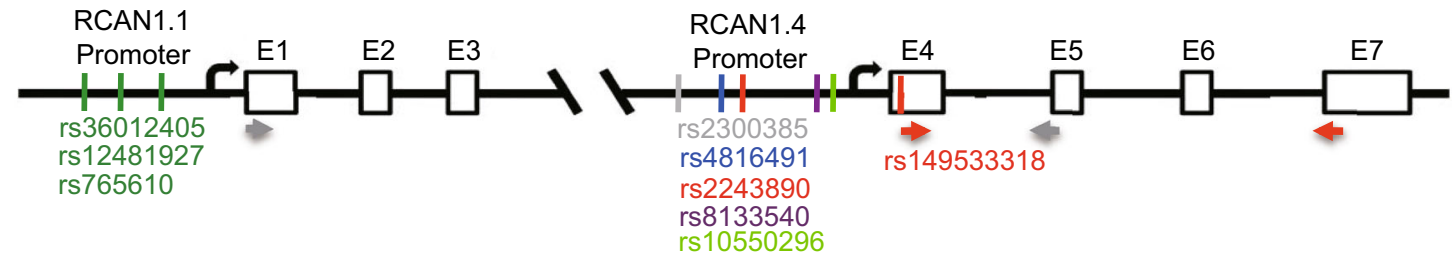

B

E10.5 mouse embryo

Hearts

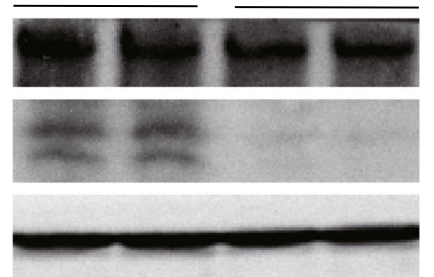

D

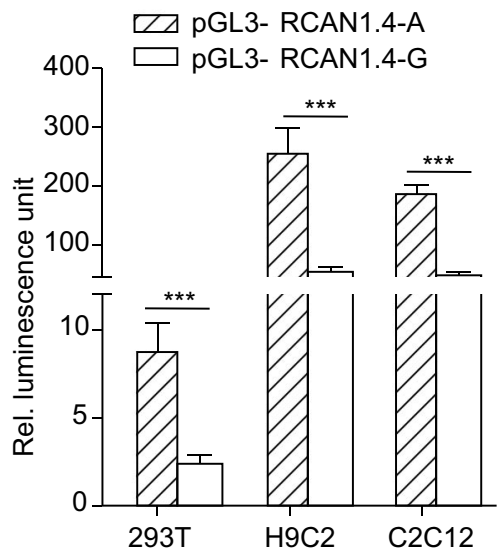

C

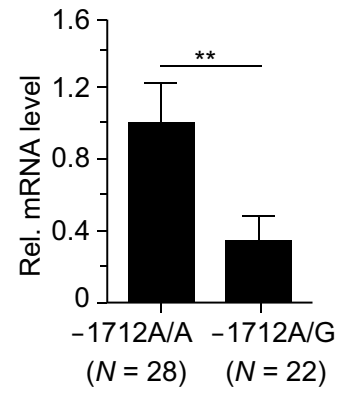

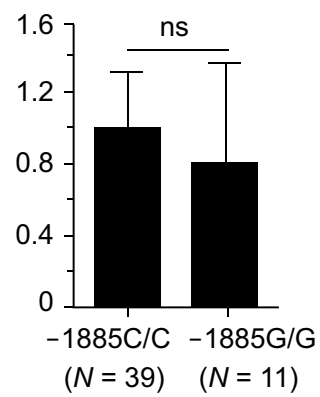

E

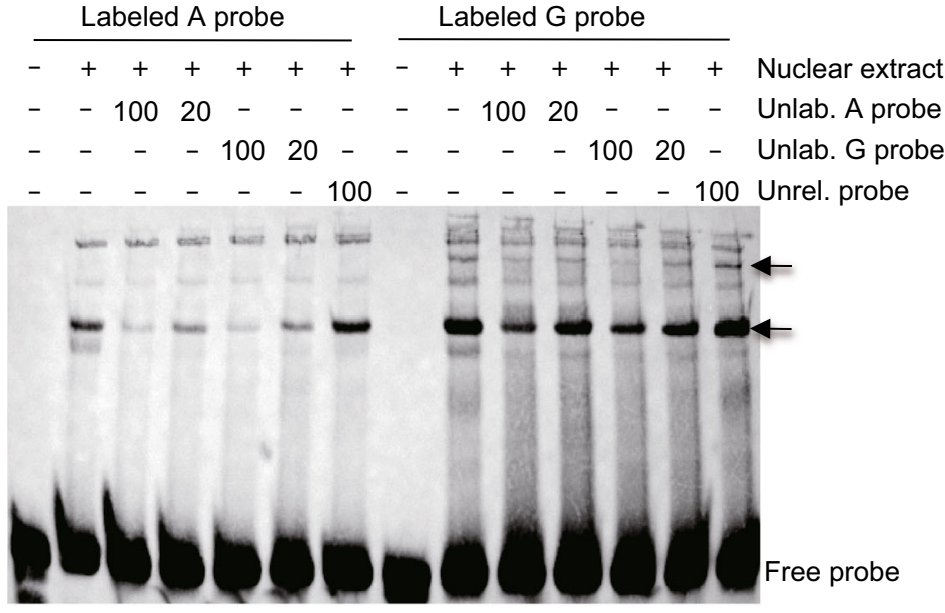

$\mathrm{F}$

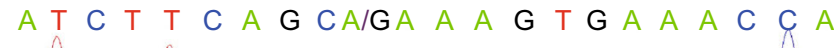

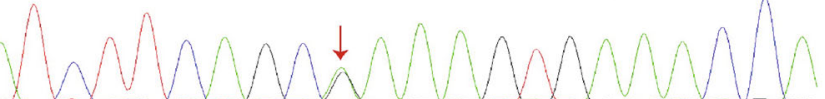

G

pGL3

pGL3-NFAT

$\triangle$ pGL3-NFAT+RCAN1.4

ШD pGL3-NFAT+RCAN1.4 E25K $^{2}$

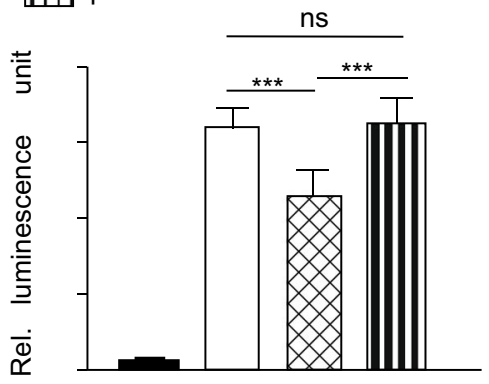

$\mathrm{H}$

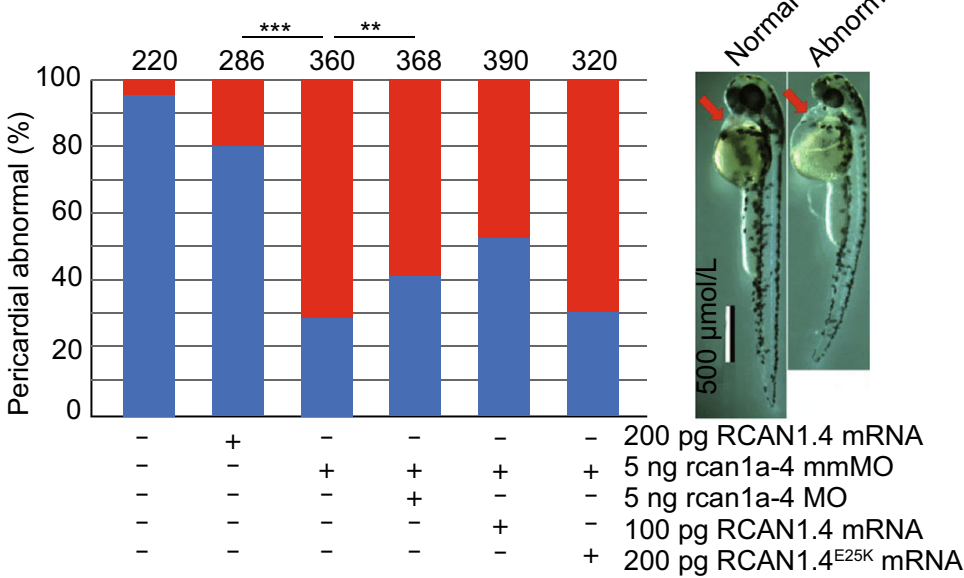


Figure 1. Decreased activity of RCAN1.4 is a potential risk factor for congenital heart disease. (A) Schematic structural diagrams of the human RCAN1 gene and the location of eight SNPs of RCAN1 identified in this study. Black arrows represent the transcription start sites of RCAN1.1 and RCAN1.4. Grey and red arrows represent the qPCR primers for RCAN1.1 and RCAN1.4, respectively. (B) Western blot of RCAN1.1 and RCAN1.4 proteins in mouse embryonic hearts and heads at embryonic day 10.5 (E10.5) and in a human foetal heart (20 weeks). $\beta$-Actin was used as a loading control. The images shown are representative of three experiments with similar results. (C) The minor $G$ allele of rs2243890 attenuates the expression of RCAN1.4 in human heart samples from sporadic CHD patients. QRT-PCR analysis of RCAN1.4 mRNA expression in sporadic CHD patients with $-1,712 \mathrm{~A} / \mathrm{A}$ or $\mathrm{A} / \mathrm{G}$ alleles of rs2243890 in RCAN1 gene (left panel) and with $-1,885 \mathrm{C} / \mathrm{C}$ or $\mathrm{G} / \mathrm{G}$ alleles of $\mathrm{rs} 2300385$ (right panel). The numbers of human heart samples used for QRT-PCR for each genotype were given under each column. GAPDH was used as an internal control. (D) The minor $G$ allele of rs2243890 reduces the activity of the RCAN1.4 promoter in an in vitro luciferase reporter assay. The effects of the minor $\mathrm{G}$ and major A alleles of rs2243890 on the promoter activity of RCAN1.4 were separately analyzed in the human embryo kidney cells (293T), the rat cardiomyocytederived cells $(\mathrm{H} 9 \mathrm{C} 2)$ and the mouse myoblasts (C2C12) using dual-reporter luciferase system. Values represent the means \pm SEM of three separate experiments. Non-parametric tests were used for statistical analysis of the results in (D). (E) The minor $G$ allele probe of rs 2243890 displays a higher binding affinity for unknown nuclear proteins from a nuclear extract of 293T cells. The results of an EMSA performed by incubating biotin-labelled minor $G$ and major $A$ allelic probes ( $G$ probe and $A$ probe, respectively) of rs2243890 with the nuclear extract from HEK 293T cells in the presence of unlabelled competitor ( $G$ probe and $A$ probe, respectively) or non-competitor (unrelated probe) probes. The first arrow indicates a weak, but specific shift band only for labeled $\mathrm{G}$ probe. The second arrow indicates a strong shift band with higher affinity to $G$ probe than to $A$ probe. The numbers above the image are the quantity $(\mathrm{pm})$ of unlabeled probes or unrelated probe used for the assay. The Image shown is representative of three experiments with similar results. (F) Sanger sequencing results showing a heterozygous mutation (A/G) at $n t 75$ (red arrow) in RCAN1.4. (G) Statistical analyses of a luciferase reporter assay in 293T cells transfected with plasmids as indicated, which revealed that this rare mutation abolishes the suppression of RCAN1.4 on NFAT-mediated transcription. $(\mathrm{H})$ The RCAN1.4 ${ }^{\mathrm{E} 25 \mathrm{~K}}$ mutant is a loss-of-function mutation in zebrafish. Right panel: zebrafish embryos with normal or enlarged pericardium after injection of $5 \mathrm{ng}$ rcan1a-4 MO. Red arrows indicate the paricardial cavity of zebrafish embryoes. Left panel: the response frequencies of pericardial defects. Co-injection of $100 \mathrm{pg}$ or $200 \mathrm{pg}$ of wt RCAN1.4 mRNA partially suppressed rcan1a-4 MO-mediated CHD phenotype (enlarged pericardium) while co-injected $200 \mathrm{pg}$ RCAN1.4 ${ }^{\mathrm{E} 25 \mathrm{~K}} \mathrm{mRNA}$ has no effect at all. The number above each bar is the total number of embryos examined under each experimental condition. $P$ value was calculated by $X^{2}$ analysis. ${ }^{\star} P<0.05,{ }^{\star \star} P<0.01,{ }^{* \star \star} P<0.001$, ns: not significant. calculation (version 3.1.2, http://biostat.mc.vanderbilt.edu/ wiki/Main/PowerSampleSize).

A further stratified analysis of rs2243890 A>G was performed based on different subtypes of $\mathrm{CHDs}$. The most significant differences in $\mathrm{CHD}$ vs. control were observed in an additive genetic model in septal defects, which had a 1.51-fold higher risk in sporadic CHD patients $(\mathrm{OR}=1.51$, $95 \% \mathrm{Cl}=1.11-2.07, P=0.01$ ) and conotruncal defects, which had a 2.18 -fold higher $(\mathrm{OR}=2.18,95 \% \mathrm{Cl}=1.59$ 2.99, $P=1.33 \mathrm{E}-06$ ) risk in sporadic $\mathrm{CHD}$ patients (Table S3). There were no significant associations observed between rs2243890 A>G and other CHD subtypes, including right ventricular outflow tract obstruction (RVOTO) $(P=0.12)$, left ventricular outflow tract obstruction (LVOTO) $(P=0.34)$ and patent ductus arteriosus (PDA) $(P=0.41)$ (Table S3).

Since the minor $\mathrm{G}$ allele of rs2243890 (at $-1,712 \mathrm{bp}$ ) was located in the promoter region for RCAN1.4 transcript, we hypothesized that the $\mathrm{G}$ allele may contribute to the risk of CHD by influencing RCAN1.4 transcription. To test our hypothesis, we evaluated the RCAN1.4 mRNA level in human heart tissue samples from sporadic CHD patients by qRT-PCR. We found that RCAN1.4 mRNA level in human heart samples with heterozygous $A / G$ alleles was almost threefold lower than that of samples with homozygous A/A alleles (Fig. 1C), while as a control, the difference in RCAN1.4 mRNA level between the heart samples carrying either heterozygous $C / G$ alleles or homozygous $G / G$ alleles of rs2300385 (at $-1,885$ bp) was not significant (Fig. 1C). These results suggest that the minor $\mathrm{G}$ allele of rs2243890 in RCAN1 may affect CHD by decreasing rather than enhancing the transcription of RCAN1.4. To further confirm the effect of rs2243890 $A>G$, we evaluated the RCAN1.4 promoter activity of the $A$ and $G$ alleles of rs 2243890 using a dual-luciferase reporter assay in HEK293, H9C2 and C2C12 cells. The results showed that the transcriptional activity of the $G$ allele was significantly lower than that of the $A$ allele in all three cell lines (Fig. 1D), demonstrating that the $G$ allele of rs2243890 attenuates RCAN1.4 transcription. Taken together, these results suggest that the minor $G$ allele of rs2243890 in RCAN1 may contribute to $\mathrm{CHD}$ by inhibiting rather than enhancing RCAN1.4 transcription.

As the minor $\mathrm{G}$ allele of rs2243890 decreased RCAN1.4 promoter activity (Fig. 1D), we questioned whether these changes might be due to its different binding affinity for some transcription factors. Using a major probe and a minor probe containing the A allele and $\mathrm{G}$ allele, respectively (Table S5), we performed an EMSA to evaluate the binding affinity of these alleles for unknown transcription factors in 293T cells. The results showed that the $G$ allele displayed a higher binding affinity for some nuclear protein(s) than the $A$ allele did (Fig. 1E). As the minor $G$ allele decreased RCAN1.4 promoter activity compared with the major $A$ allele, we inferred that the specific binding protein(s) probably function as transcriptional repressors. 
Table 1. Association of SNP rs2243890 A>G in RCAN1.4 promoter region with CHDs in two independent case-control studies

\begin{tabular}{|c|c|c|c|c|c|c|c|c|}
\hline \multirow{2}{*}{ Study } & \multicolumn{2}{|c|}{ Genotype $^{a}$} & \multicolumn{2}{|c|}{ MAF } & \multirow{2}{*}{ Genetic model } & \multirow{2}{*}{ OR $(95 \% \mathrm{Cl})^{\mathrm{b}}$} & \multirow{2}{*}{$P$-value ${ }^{b}$} & \multirow{2}{*}{$\begin{array}{c}P^{\circ}, \mathrm{HWE} \\
\text { test }\end{array}$} \\
\hline & Control & Case & Control & Case & & & & \\
\hline \multirow{4}{*}{$\begin{array}{l}\text { Shandong } \\
\text { samples }\end{array}$} & \multirow{4}{*}{$1 / 20 / 192$} & \multirow{4}{*}{$1 / 76 / 335$} & \multirow{4}{*}{0.05} & \multirow{4}{*}{0.09} & Codominant & NA & 0.010 & \multirow{4}{*}{0.44} \\
\hline & & & & & Dominant & $2.11(1.26-3.53)$ & 0.004 & \\
\hline & & & & & Recessive & $0.52(0.03-8.38)$ & 0.644 & \\
\hline & & & & & Additive & $1.98(1.20-3.27)$ & 0.007 & \\
\hline \multirow{4}{*}{$\begin{array}{l}\text { Shanghai } \\
\text { samples }\end{array}$} & \multirow{4}{*}{ 6/129/972 } & \multirow{4}{*}{ 3/83/372 } & \multirow{4}{*}{0.06} & \multirow{4}{*}{0.10} & Codominant & NA & 0.004 & \multirow{4}{*}{0.44} \\
\hline & & & & & Dominant & $1.65(1.23-2.22)$ & 8.77-E04 & \\
\hline & & & & & Recessive & $1.15(0.29-4.64)$ & 0.840 & \\
\hline & & & & & Additive & $1.57(1.19-2.07)$ & 0.002 & \\
\hline \multirow{4}{*}{$\begin{array}{l}\text { Combined- } \\
\text { analysis }\end{array}$} & \multirow{4}{*}{$7 / 149 / 1,164$} & \multirow{4}{*}{$4 / 159 / 707$} & \multirow{4}{*}{0.06} & \multirow{4}{*}{0.10} & Codominant & NA & $2.98 \mathrm{E}-05$ & \multirow{4}{*}{0.34} \\
\hline & & & & & Dominant & $1.76(1.36-2.27)^{d}$ & $1.71 \mathrm{E}-05^{\mathrm{d}}$ & \\
\hline & & & & & Recessive & $0.98(0.28-3.41)^{d}$ & $0.980^{d}$ & \\
\hline & & & & & Additive & $1.66(1.30-2.11)^{d}$ & $4.68 \mathrm{E}-05^{\mathrm{d}}$ & \\
\hline
\end{tabular}

By Sanger sequencing we also confirmed the CHDspecific rare mutation RCAN1.4 $4^{\mathrm{E} 25 \mathrm{~K}}$ identified from targetcapture sequencing (Fig. 1F). The MAF for RCAN1.4 ${ }^{\mathrm{E} 25 \mathrm{~K}}$ in the ExAC database was as rare as $3.35 \mathrm{E}-05$ (4/119506). To assess whether this RCAN1.4 ${ }^{\mathrm{E} 25 \mathrm{~K}}$ mutant plays a role in CHD development, we performed an NFAT promoter-mediated luciferase reporter assay. Results showed that mutant RCAN1.4 ${ }^{\mathrm{E} 25 \mathrm{~K}}$ significantly impaired suppression of NFATmediated transcription (Figs. $1 \mathrm{G}$ and $\mathrm{S} 1 \mathrm{~B}$ ), suggesting that RCAN1.4 ${ }^{\mathrm{E} 25 \mathrm{~K}}$ may be a loss-of-function mutant.

The E25 of human RCAN1.4 protein is conserved from the zebrafish to human (Fig. S1C). About $70 \%$ of zebrafishes injected with antisense morpholino-modified oligonucleotides (MO) for zebrafish rcan1a-4 (a zebrafish homolog of human RCAN1.4) (Alghanem et al., 2017) displayed an enlarged pericardium (a CHD phenotype) and this rcan1a-4 MO-mediated CHD phenotype could be partially rescued by co-injected human RCAN1.4 mRNA in a dosage-dependent manner (Fig. $1 \mathrm{H}$ ). However, human RCAN1.4 $4^{\mathrm{E} 25 \mathrm{~K}}$ mRNA failed to rescue rcan1a-4 MO-mediated CHD phenotype, suggesting again that RCAN1.4 ${ }^{\mathrm{E} 25 \mathrm{~K}}$ is a loss-of-function mutation in vivo.

In this study, the SNP rs2243890 in RCAN1.4 promoter region was found to be significantly associated with $\mathrm{CHD}$ and further validated to be significantly associated with septal and conotruncal defects of CHD by analysing blood samples from 870 sporadic CHD patients and 1,320 normal controls from a Han Chinese population. Our results, therefore, adds one more piece of evidence supporting the conclusion that genetic associations with $\mathrm{CHD}$ have a high phenotypic specificity (Soemedi et al., 2012; Cordell et al., 2013) and also suggest a population specificity for genetic association of SNP rs2243890 with CHD because reported GWAS studies did not find the association of SNP rs2243890 with CHD in Caucasian (Cordell et al., 2013).
Consistent with the results from two previous genetic association studies (Guo et al., 2015; Li et al., 2015), we confirmed that three other SNPs (rs765610, rs12481297 and rs36012405) in the RCAN1.1 promoter region were also not associated with CHD. Our Western blot analyses further showed that the RCAN1.4 protein was expressed in both mouse and human embryonic hearts and the RCAN1.4, but not RCAN1.1, protein level was differentially up-regulated in developing mouse embryonic hearts (Fig. 1B). These results support the notion that RCAN1.4, but not RCAN1.1, may contribute to CHD pathogenesis in the Han Chinese population.

RCAN1, also known as Down syndrome candidate region 1 (DSCR1), is located in syntenic regions of human chromosome 21 . Approximately $40 \%-60 \%$ of DS patients are accompanied by CHD (Stoll et al., 1998; Vis et al., 2009; Elmagrpy et al., 2011). RCAN1 triplication was thought to be a potential cause for CHD in DS (Lange et al., 2004). However, Lyle et al. identified eight new DS cases of partial monosomy 21 . One patient carried only a $1.48 \mathrm{Mb}$ deletion containing eight genes, including RCAN1, and displayed a relatively severe DS phenotype, including cardiac anomaly (Lyle et al., 2009). Now, our study revealed that rs2243890 $A>G$ in the RCAN1.4 promoter increases the risk of $\mathrm{CHD}$ by decreasing RCAN1.4 transcription. We not only demonstrated that the minor $G$ allele of rs2243890 significantly decreased RCAN1.4 transcription but also showed that the rare mutation RCAN1.4 ${ }^{\mathrm{E} 25 \mathrm{~K}}$ identified in a VSD patient almost completely abolished RCAN1.4 activity in both in vivo zebrafish model as well as in an in vitro luciferase reporter assay. Altogether, these studies suggest that the expression or activity of RCAN1, rather than the RCAN1 copy number, may contribute to DS-associated CHD. Our study may provide a new understanding of the role of the RCAN1 gene in human $\mathrm{CHD}$. 


\section{FOOTNOTES}

This work was supported by the grants from National Key Research and Development Program of China (2016YFC1000500) to Hongyan Wang and Wufan Tao, the National Natural Science Foundation of China (Grant Nos. 31671330 and 31471380) to Wufan Tao and (81472050) to Xueyan Yang, the National Natural Science Foundation of China (Grant Nos. 81430005 and 31521003) and the Commission for Science and Technology of Shanghai Municipality (13JC1407600) to Hongyan Wang.

Liangping Cheng, Peiqiang Li, He Wang, Xueyan Yang, Huiming Zhou, Wufan Tao, Jie Tian and Hongyan Wang declare that they have no conflict of interest.

All procedures followed were in accordance with the ethical standards of the responsible committee on human experimentation (institutional and national) and with the Helsinki Declaration of 1975, as revised in 2000 (5). Informed consent was obtained from all patients for being included in the study. Additional informed consent was obtained from all patients for which identifying information is included in this article. All institutional and national guidelines for the care and use of laboratory animals were followed.

Liangping Cheng ${ }^{1,2}$ (D), Peiqiang Li ${ }^{3,4}$, He Wang ${ }^{4}$, Xueyan Yang ${ }^{4}$, Huiming Zhou ${ }^{1}$, Wufan Tao ${ }^{2,5 凶}$, Jie $\operatorname{Tian}^{1 凶}$, Hongyan Wang ${ }^{2,4 \bowtie}$ (D)

${ }^{1}$ Key Laboratory of Pediatrics in Chongqing, Chongqing International Science and Technology Cooperation Center for Child Development and Disorders, Department of Cardiovascular Medicine, Children's Hospital of Chongqing Medical University, Chongqing 400014, China

2 Obstetrics Gynecology Hospital, The Institute of Reproduction and Developmental Biology, Fudan University, Shanghai 200011, China

${ }^{3}$ Institute of Genetics, School of Basic Medical Sciences, Lanzhou University, Lanzhou 730000, China

4 The State Key Laboratory of Genetic Engineering, MOE Key Laboratory of Contemporary Anthropology, Collaborative Innovation Center of Genetics and Development, School of Life Science, Fudan University, Shanghai 200433, China

${ }^{5}$ Institute of Developmental Biology and Molecular Medicine, Fudan University, Shanghai 200433, China

$\triangle$ Correspondence: wufan_tao@fudan.edu.cn (W. Tao), jietian@cqmu.edu.cn (J. Tian), wanghy@fudan.edu.cn ( $\mathrm{H}$. Wang)

\section{OPEN ACCESS}

This article is distributed under the terms of the Creative Commons Attribution 4.0 International License (http://creativecommons.org/ licenses/by/4.0/), which permits unrestricted use, distribution, and reproduction in any medium, provided you give appropriate credit to the original author(s) and the source, provide a link to the Creative Commons license, and indicate if changes were made.

\section{REFERENCES}

Alghanem AF, Wilkinson EL, Emmett MS, Aljasir MA, Holmes K, Rothermel BA, Simms VA, Heath VL, Cross MJ (2017) RCAN1.4 regulates VEGFR-2 internalisation, cell polarity and migration in human microvascular endothelial cells. Angiogenesis 20:341358

Cordell HJ, Bentham J, Topf A, Zelenika D, Heath S, Mamasoula C, Cosgrove C, Blue G, Granados-Riveron J, Setchfield K et al (2013) Genome-wide association study of multiple congenital heart disease phenotypes identifies a susceptibility locus for atrial septal defect at chromosome 4p16. Nat Genet 45:822-824

de la Pompa JL, Timmerman LA, Takimoto H, Yoshida H, Elia AJ, Samper E, Potter J, Wakeham A, Marengere L, Langille BL et al (1998) Role of the NF-ATc transcription factor in morphogenesis of cardiac valves and septum. Nature 392:182-186

Elmagrpy Z, Rayani A, Shah A, Habas E, Aburawi EH (2011) Down syndrome and congenital heart disease: why the regional difference as observed in the Libyan experience? Cardiovasc $\mathrm{J}$ Afr 22:306-309

Fuentes JJ, Pritchard MA, Planas AM, Bosch A, Ferrer I, Estivill X (1995) A new human gene from the down syndrome critical region encodes a proline-rich protein highly expressed in fetal brain and heart. Hum Mol Genet 4:1935-1944

Guo RY, Li XF, Bai S, Guo J, Ding N, Li ZZ (2015) Association between DSCR1 variations and congenital heart disease susceptibility. Med Sci Monit 21:3536-3539

Lange AW, Molkentin JD, Yutzey KE (2004) DSCR1 gene expression is dependent on NFATc1 during cardiac valve formation and colocalizes with anomalous organ development in trisomy 16 mice. Dev Biol 266:346-360

Lange AW, Yutzey KE (2006) NFATc1 expression in the developing heart valves is responsive to the RANKL pathway and is required for endocardial expression of cathepsin K. Dev Biol 292:407-417

Li X, Wang G, An Y, Li H, Li Y, Wu C (2015) Association between sequence variations in RCAN1 promoter and the risk of sporadic congenital heart disease in a Chinese population. Pediatr Cardiol 36:1393-1399

Lyle R, Bena F, Gagos S, Gehrig C, Lopez G, Schinzel A, Lespinasse J, Bottani A, Dahoun S, Taine L et al (2009) Genotype-phenotype correlations in Down syndrome identified by array $\mathrm{CGH}$ in 30 cases of partial trisomy and partial monosomy chromosome 21. Eur J Hum Genet 17:454-466

Qin L, Zhao D, Liu X, Nagy JA, Hoang MV, Brown LF, Dvorak HF, Zeng $H$ (2006) Down syndrome candidate region 1 isoform 1 mediates angiogenesis through the calcineurin-NFAT pathway. Mol Cancer Res 4:811-820

Soemedi R, Topf A, Wilson IJ, Darlay R, Rahman T, Glen E, Hall D, Huang N, Bentham J, Bhattacharya S et al (2012) Phenotypespecific effect of chromosome 1q21.1 rearrangements and GJA5 duplications in 2436 congenital heart disease patients and 6760 controls. Hum Mol Genet 21:1513-1520

Stoll C, Alembik Y, Dott B, Roth MP (1998) Study of Down syndrome in 238,942 consecutive births. Ann Genet 41:44-51 
Vis JC, Duffels MG, Winter MM, Weijerman ME, Cobben JM, Huisman SA, Mulder BJ (2009) Down syndrome: a cardiovascular perspective. J Intellect Disabil Res 53:419425
Yang J, Rothermel B, Vega RB, Frey N, McKinsey TA, Olson EN, Bassel-Duby R, Williams RS (2000) Independent signals control expression of the calcineurin inhibitory proteins MCIP1 and MCIP2 in striated muscles. Circ Res 87:E61-E68

Liangping Cheng and Peiqiang Li have contributed equally to this work.

Electronic supplementary material The online version of this article (https://doi.org/10.1007/s13238-018-0525-8) contains supplementary material, which is available to authorized users. 